\title{
Arcjet Thruster Operated With Different Propellants
}

\author{
Heji Huang, Wenxia Pan, and Chengkang Wu
}

\begin{abstract}
As a simple and reliable propulsion system, arcjet thrusters have been used in multiple satellite missions. The performance of an arcjet thruster is controlled by the characteristics of the arc-discharge behavior and the arc-electrode interactions to a large extent. The nozzle of an arcjet thruster is where the input electric energy is converted into kinetic energy. The nozzle images of an arcjet thruster operated with different propellants are presented in this paper.
\end{abstract}

Index Terms-Arcjet thruster, electric propulsion, temperature measurement.

C OMPARED with conventional chemical-propulsion methods such as chemical rockets, electric-propulsion systems are more advanced, with the higher specific impulse $I_{\mathrm{sp}}$, higher thrust efficiency, better controllability, longer operating duration, etc. [1], [2] According to the Tsiolkovsky rocket equation, higher $I_{\mathrm{sp}}$ is favorable for increasing the ratio of payload mass to total launch mass. However, from the view of energy consumption, an optimized $I_{\mathrm{sp}}$ value is needed to balance the total power requirement and the propellant consumption for certain space missions where the specific velocity change of the vehicle $\Delta V$ is needed. A propulsion system with very high $I_{\mathrm{sp}}$, e.g., an ion thruster, is usually expected for high- $\Delta V$ missions such as deep-space explorations. On the other hand, for some satellite missions including north-south station keeping and orbital maneuvering, an arcjet thruster with moderate $I_{\mathrm{sp}}$ and a relatively larger thrust level is preferred.

An arcjet thruster uses an arc discharge to heat the injected propellant. With a de Laval nozzle, the heated propellant is further accelerated and exhausted to generate the required thrust. The performance of the arcjet thruster is controlled by the characteristics of the arc-discharge behavior and the arc-electrode interactions to a large extent. Generally, the anode of arcjet thrusters also acts as the de Laval nozzle, which makes it a key component. It is in the anode/nozzle that the conversion of thermal energy into directed kinetic energy occurs.

The outside and inside nozzle images of a $1 \mathrm{~kW}$ arcjet thruster developed in the Institute of Mechanic, Chinese Academy of Sciences were taken using a Sony DSR-PD198P

Manuscript received December 1, 2010; revised April 17, 2011; accepted May 6, 2011. Date of publication June 2, 2011; date of current version November 9, 2011. This work was supported by the National Natural Science Foundation of China under Grant 50836007 and Grant 10921062.

The authors are with the Institute of Mechanics, Chinese Academy of Sciences, Beijing 100190, China.

Color versions of one or more of the figures in this paper are available online at http://ieeexplore.iee.org.

Digital Object Identifier 10.1109/TPS.2011.2155097 video camera. The outside nozzle temperature was measured by two infrared pyrometers with a combined measuring range of $473-2273 \mathrm{~K}$. A $45^{\circ}$ tilted copper mirror was used to reflect the end-on view of the nozzle to the outer camera. Argon, nitrogen, hydrogen, and the mixture of nitrogen-hydrogen with a volume ratio of $1: 2$ were used as the propellant, respectively. When the arcjet thruster is working, to avoid oversaturation caused by the strong arc and plume emissions, a 698-nm interference filter was used to cut off the line emissions of nitrogen or hydrogen plasmas, so that only thermal radiation is recorded in the inner nozzle images. The nozzle of the arcjet thruster is cooled regeneratively by forcing the cold propellant to pass through the outside surface of the hot nozzle before entering the convergent section and being arc heated. The nozzle has a $\Phi 0.7-\mathrm{mm}$ throat with an exit diameter of $11 \mathrm{~mm}$.

Fig. 1(a) to (d) show the outside nozzle images using Ar, $\mathrm{N}_{2}, \mathrm{H}_{2}$, and $\mathrm{N}_{2}-\mathrm{H}_{2}$ as the propellant, respectively. The arc current for all the cases is fixed at $8 \mathrm{~A}$. However, with different propellants, the arc voltage varies a lot. When the propellant was Ar with a flow rate of $133 \mathrm{mg} / \mathrm{s}$, the arc voltage was only $25 \mathrm{~V}$, which leads to a lower enthalpy of the jet, and the nozzle outside temperature was as low as $760 \mathrm{~K}$ at a stable working condition. The temperature-measurement spots are the same for all the figures as marked by the white circle in Fig. 1(a). When the propellants were $\mathrm{N}_{2}, \mathrm{H}_{2}$, or $\mathrm{N}_{2}-\mathrm{H}_{2}$, the arc voltage increased to 99,109 , and $101 \mathrm{~V}$, respectively, leading to higher nozzle temperatures of 1220,1600 , and $1700 \mathrm{~K}$. A markedly nozzle-temperature increase was observed when hydrogen was added because of the high thermal conductivity of hydrogen.

In the case of inside nozzle images, although arc and plume exist during the arcjet-thruster operation, the 698-nm interference filter can effectively remove the emission lines from them when the propellant is $\mathrm{N}_{2}, \mathrm{H}_{2}$, or $\mathrm{N}_{2}-\mathrm{H}_{2}$. Based on this, thermal-radiation signals of the inner nozzle surface can be considered to be a kind of near infrared images. With careful calibrations, these images can be used to reveal quantitative inner-nozzle-temperature distributions. Relevant work is now in progress. The high temperature zone represented by higher image intensity in the inside nozzle images is mainly caused by the arc root attachment,where Joule heat remarkably increases the surface temperature. And the width of the high temperature zone can reveal the degree of constriction of the arc root attachment qualitatively. Preliminary results show that no matter what kind of propellants were used, the high-temperature zone stays near the exit of the throat at a stable working condition, indicating that the position of arc-root attachment is also on the surface of the nozzle-expanding section near the throat exit. Results also show that the propellant compositions affect the arc-root attachment remarkably. When the outside nozzle 


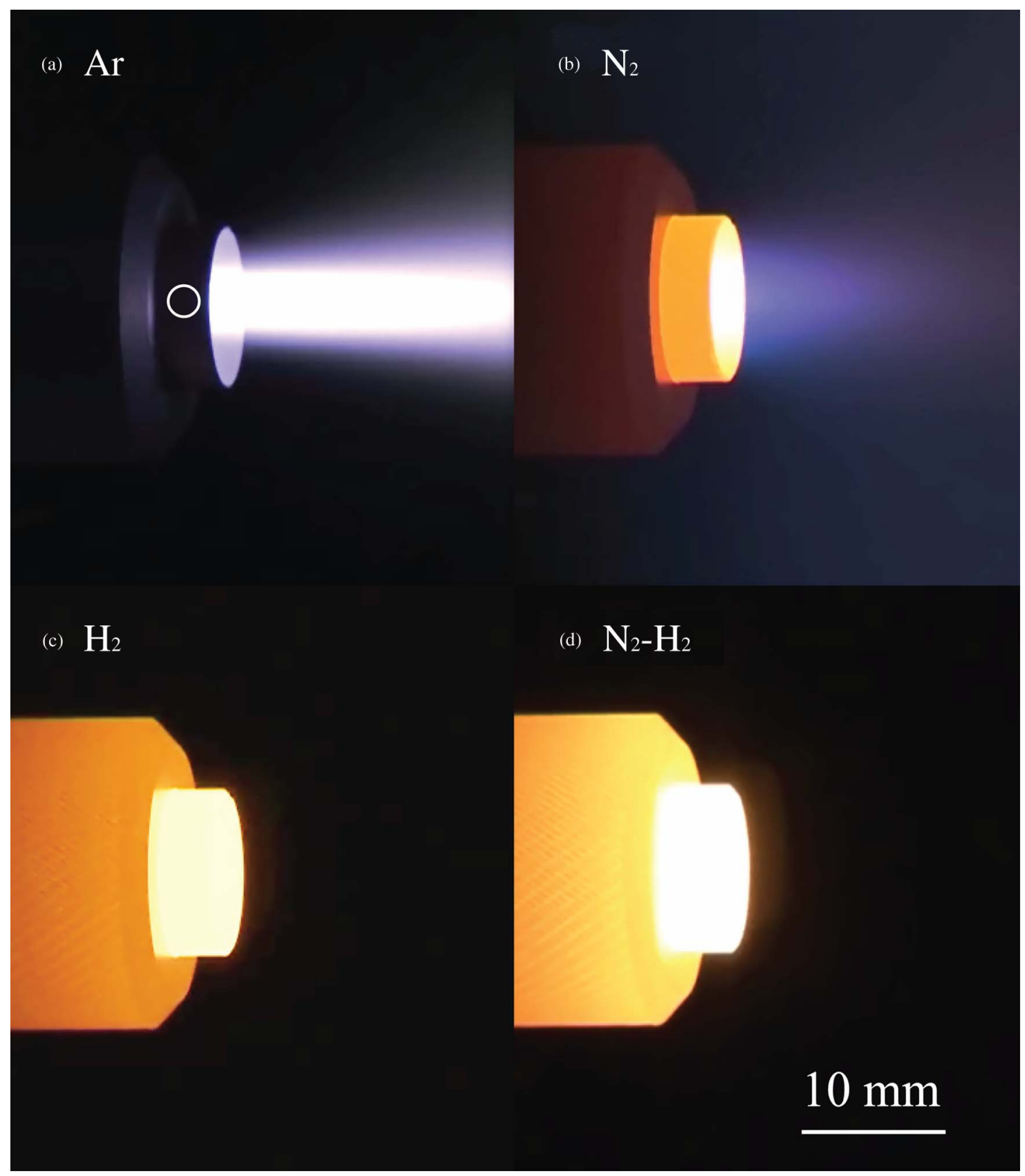

Fig. 1. Outside nozzle images of the arcjet thruster with different propellants at stable working condition. (a) Pure argon with a flow rate of $133 \mathrm{mg} / \mathrm{s}$. (b) Pure nitrogen with a flow rate of $83 \mathrm{mg} / \mathrm{s}$. (c) Pure hydrogen with a flow rate of $12 \mathrm{mg} / \mathrm{s}$. (d) nitrogen-hydrogen mixture with a total flow rate of $36 \mathrm{mg} / \mathrm{s}$. The white circle marked in (a) represents the temperature-measurement spot.

temperatures are similar, the arc-root-attachment area is much narrower when pure $\mathrm{H}_{2}$ was used.

In summary, outside and inside nozzle images of the arcjet thruster operated with different propellants were taken in this paper, which might give some indication of different arcing and heat-transfer behavior with these propellants.

\section{REFERENCES}

[1] G. Saccoccia and W. Berry, "European electric propulsion activities and programmes," Acta Astron., vol. 47, no. 2-9, pp. 193-203, Jul.-Nov. 2000.

[2] G. W. Butler and R. J. Cassady, "Directions for arcjet technology development," J. Propulsion Power, vol. 12, no. 6, pp. 1026-1034, Nov./Dec. 1996 